\title{
Mean ST Segment Duration
}

National Cancer Institute

\section{Source}

National Cancer Institute. Mean ST Segment Duration. NCI Thesaurus. Code C62145.

The duration (time) connecting the QRS complex and the T wave. The ST segment starts at the J point and ends at the beginning of the $T$ wave. 